\title{
Introduction to Chapter 3
}

Here, we move from the very grounded, synthetic Latin American farmer participants' experience of action research in Chapter 2 to that of researchers collaborating within an agricultural development project designed to use participatory action research (PAR). Mutimukuru-Maravanyika and her co-authors reflexively examine their own experience of carrying out PAR within a complex institutional framework, spanning divergent countries, disciplines, cultures and research organizations.

Using examples of conflict, miscommunication and divergent goals to illustrate problems that often bedevil attempts to work across disciplines - something needed in any ACM programme - these authors are able to extract recurrent patterns and offer the solutions they were able to devise within the "Aquatic Agricultural Systems (AAS) Program" in the Barotse Floodplain in western Zambia. Practitioners of ACM will recognize many of the issues discussed in this chapter, issues that often plague interdisciplinary approaches, regardless of the natural resource system studied.

This chapter also highlights the importance of recognizing the key roles of researchers themselves (ourselves) as co-producers of knowledge, as collaborators with communities and among themselves. We study and work with communities and other stakeholders but must also attend to the structures, power, learning and collaboration processes within our teams if we hope to work effectively. This example explores a complex project in which the PAR approach was formally institutionalized; it draws from experience in a very complex and structured programme.

One of the lessons we have learned from our authors' experience (see e.g., Chapter 6) is the necessity to devote more attention to making sure ACM teams and communities better understand the basics - the principles of the ACM approach - than we originally realized. 